\title{
Elongación esofágica y esofagoplastía temprana en neonatos de alto riesgo con atresia tipo long gap
}

\author{
Rogelio Sancho-Hernández, * ${ }^{*}$ Lizbeth Solorio-Rodríguez, ${ }^{*}$ Kevin Omar Ham-Armenta, \\ Yolanda Iribe-Gaxiola, Francisco Javier Cuevas-Schacht*
}

*Instituto Nacional de Pediatría, Ciudad de México; ${ }^{\star}$ Hospital Materno Perinatal Mónica Pretelini Sáenz,

Ciudad de Toluca, Estado de México.

Trabajo recibido: 23-X-2014; aceptado: 08-II-2015

\begin{abstract}
RESUMEN. Introducción: La atresia de esófago con brecha amplia (long gap) se emplea como sinónimo de atresia esofágica tipo I, pero para efectos operativos son todas aquéllas que independientemente de su tipo imposibilitan la anastomosis primaria. La técnica de elongación esofágica externa (procedimiento de Foker) provee una opción de anastomosis temprana. No existe en la literatura nacional alguna experiencia reportada con esta técnica, hay pocos estudios que describen la eficacia y seguridad en el período neonatal. Descripción de los casos: Dos prematuros, ambos con síndrome de Down y atresia esofágica tipo long gap de $4.5 \mathrm{~cm}$ y 6 cuerpos vertebrales, respectivamente. En ambos pacientes se practicó elongación esofágica externa con 4 y 7 días de tracción y se consiguió la anastomosis primaria a los 33 y 37 días de vida. Discusión: La técnica de Foker permitió en nuestros neonatos alcanzar longitudes suficientes para concebir la anastomosis temprana en las atresias de esófago tipo long gap. Con una modificación del método original, la técnica puede representar una opción para esta conducta de esofagoplastía por etapas en los recién nacidos de alto riesgo con prematurez y cromosomopatías con complicaciones menores a otras esofagoplastías tardías.
\end{abstract}

Palabras clave: Elongación esofágica, tracción externa, prematuros.

ABSTRACT. Introduction: Atresia of the esophagus with long gap is used as a synonym for esophageal atresia type I, but for operational purposes are all those that, regardless of their type impossible primary anastomosis. The technique of external esophageal elongation (procedure of Foker) provides an option of early anastomosis. Does not exist in the literature any national experience reported with this technique, there are few studies that describe the efficacy and safety in newborns. Description of the cases: Two premature both with Down syndrome and esophageal atresia type long gap of $4.5 \mathrm{~cm}$ and 6 vertebral bodies, respectively; both practical esophageal elongation with 4 external and 7 days of traction and achieved primary anastomosis to the 33 and 37 days of life. Discussion: The technique of Foker allowed in our neonates reach lengths sufficient for designing the early anastomosis in the atresias of esophagus type long gap; with a modification of the original method this technique can represent an option for this conduct of esophagoplasty in stages over the newly born of high risk with prematurity and chromosomal abnormalities with minor complications to other esophagoplasties late.

Key words: Esophageal elongation, external traction, premature.

\section{INTRODUCCIÓN}

La atresia de esófago con brecha amplia (long gap), es definida como aquélla cuya distancia entre sus cabos atrésicos es mayor de $5 \mathrm{~cm}$ y se emplea como sinónimo de atresia esofágica tipo I, pero para efectos operativos, son todas aquellas atresias esofágicas que independientemente de su tipo imposibilitan la anastomosis primaria terminoterminal. Varias técnicas de elongación esofágica han sido propuestas para concebir la anastomosis temprana del esófago nativo: elongación con dilatadores, atracción magnética de los cabos atrésicos, miotomía circular y elongaciones intra o extratorácicas, con pocos estudios que aporten la eficacia y seguridad de estas técnicas durante el período neonatal. ${ }^{1}$
Tradicionalmente, el tratamiento quirúrgico de las atresias con brecha amplia corresponde a la realización de una esofagostomía cervical con gastrostomías iniciales, y posterior método quirúrgico de sustitución esofágica (esófago no nativo) con la interposición de una víscera hueca en la lactancia o infancia tardías. ${ }^{2-4}$

En los recién nacidos en quienes se ha creado una esofagostomía, la técnica de avance de Kimura es una alternativa, consiste en múltiples elongaciones extratorácicas de la derivación esofágica proximal con movilización subcutánea del estoma de manera progresiva y descendente sobre la pared anterior del tórax hasta concebir la longitud esofágica suficiente para la anastomosis tardía. Esta técnica reportada como exitosa, es-

Este artículo puede ser consultado en versión completa en http://www.medigraphic.com/neumologia 
capa del período neonatal, y el número de toracotomías y complicaciones aumentan de manera significativa. ${ }^{5}$ Contemplando que el crecimiento esofágico puede ser estimulado por fuerzas de tensión aplicado a los cabos atrésicos, la técnica de elongación esofágica de Foker provee una opción de anastomosis temprana durante la etapa neonatal en aquellas brechas amplias de 5.5 a $6.8 \mathrm{~cm}$; y aunque requiere una segunda toracotomía, el tiempo requerido entre la elongación y la anastomosis definitiva es menor que en otras técnicas descritas, minimizando la estancia prolongada y el riesgo de aspiración y complicaciones descritas para las técnicas de esofagoplastía tardías. ${ }^{6-10}$

No existe en la literatura nacional alguna experiencia reportada a través de esta técnica de elongación esofágica para conciliar la anastomosis primaria de las atresias de esófago con long gap. Se presentan dos casos clínicos y descripción en detalle de la técnica quirúrgica. Asimismo, se analizan los resultados de una revisión de la literatura de los reportes y series de casos descritos hasta el momento.

\section{MATERIAL Y MÉTODOS}

Se realizó la revisión de la literatura en inglés y español utilizando los buscadores electrónicos Medline y PubMed con los términos «atresia de esófago long gap», «elongación esofágica intra y extratorácica» y «técnica de Foker para elongación esofágica». Los resultados fueron analizados y se incluyeron las técnicas descritas de elongación esofágica que lograron mostrar utilidad en la población neonatal y la lactancia menor de tres meses de edad. Fueron excluidas las esofagoplastías tardías y técnicas de sustitución esofágicas exitosas posteriores a este grupo etario. Se propone un algoritmo para la toma de decisiones quirúrgicas.
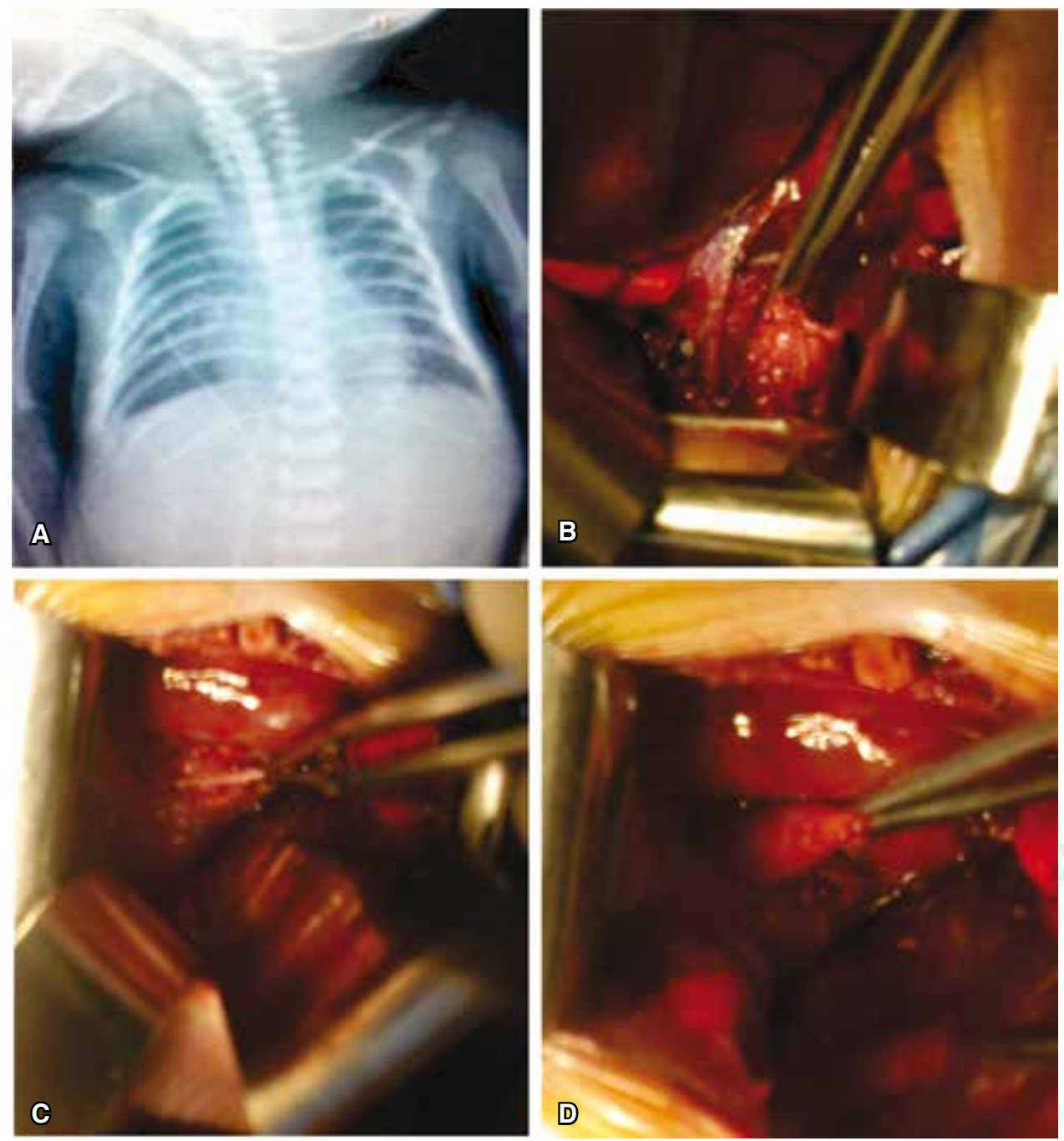

\section{Figura 1.}

A: Radiografía con sonda radiopaca en el cabo superior ciego a nivel de la cuarta vértebra torácica y ausencia de aire intestinal sugerente de atresia esofágica tipo I; B: disección y movilización del cabo superior; C: brecha amplia entre cabos atrésicos, el nervio vago en su trayecto distal permite identificar el cabo inferior; $\mathbf{D}$ : cabo distal breve y ciego de $1.5 \mathrm{~cm}$ de longitud posterior a la movilización hiatal. 



Figura 2.

A: Elongación esofágica aplicada al cabo inferior mediante un punto transflictivo y protegido por catéteres de silicona para la tracción externa; B: movilización y elongación de Foker aplicado al cabo superior; C: exteriorización de las riendas de tracción, el esófago superior marcado con un clip metálico ( ${ }^{\star}$ rojo) es sometido a tracción externa del cabo superior (TECS) y el esófago inferior (*amarillo) es elongado a través de tracción externa del cabo inferior (TECI); $\mathbf{D}$ : seguimiento radiológico de los cabos radiopacos sometidos a elongación externa, el puente entre los cabos corresponde a dos cuerpos vertebrales.

\section{DESCRIPCIÓN DE LA TÉCNICA QUIRÚRGICA}

Se procede con una mini toracotomía derecha periescapular y conservadora de músculos, se accede a través del quinto espacio intercostal a la cavidad mediastinal por un abordaje idealmente extrapleural. La ligadura y sección del cayado de la vena ácigos permite, con ayuda de la manipulación de una sonda orogástrica, la identificación del cabo esofágico superior. La disección y movilización gentil de dicho cabo permiten la máxima elongación y evita la devascularización. El cabo inferior es identificado siguiendo el curso distal del nervio vago (un gastrograma neumático transoperatorio también facilita la distensión del cabo distal); este cabo es muy breve y debe ser disecado, movilizado y medido delicadamente con ayuda de una disección hiatal diafragmática (lo cual protege la circulación arterial esofágica y diafragmática inferior). Se mide la brecha entre ambos cabos liberados, y una imposibilidad para la anastomosis primaria con cabo esofágico inferior mayor de $1 \mathrm{~cm}$ de longitud convierte el defecto en candidato a técnica de elongación externa: el cabo inferior es fijado con un punto medial, total y transflictivo en «U» a dos segmentos laterales de tubos de polivinilo (de catéteres radiopacos) con sutura no absorbible. Después, dos suturas a manera de riendas son pasadas a través de cada uno de los lúmenes de ambos tubos radiopacos de donde serán exteriorizadas para ejecutar la tracción externa. El mismo procedimiento se ejecuta para el cabo superior; ambos cabos son marcados con un clip metálico y sus respectivas riendas son exteriorizadas: el cabo superior es traccionado hacia la porción inferior de la herida (intercostal VI) y el cabo inferior hacia la porción superior de la toracotomía (intercostal IV), la tracción externa de las riendas sobre la superficie del tórax se realiza con dos pinzas protegidas con caucho para evitar la erosión sobre la piel, se fija una sonda de drenaje pleural conectada a sello de agua. A las 72 horas del posquirúrgico se inician las tracciones dinámicas de $3 \mathrm{~mm} /$ día de ambos cabos bajo sedación y analgesia neonatal. El control radiológico diario de ambos cabos radiopacos permitirá detectar las complicaciones e identificar la aproximación y contacto de los segmentos atrésicos, momento en el cual podría considerarse la esofagoplastía definitiva. 

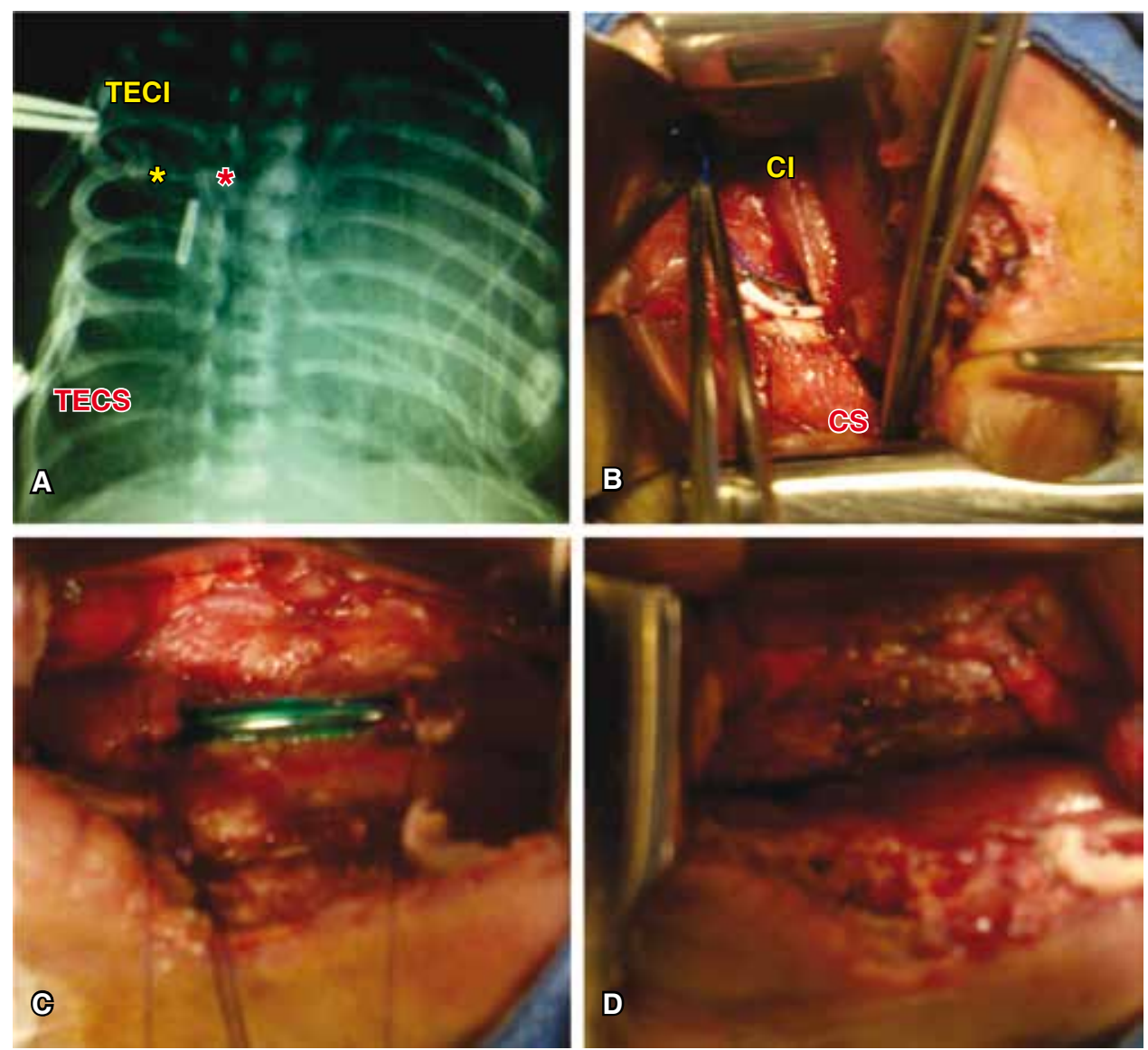

\section{Figura 3.}

A: Cabos atrésicos radiopacos que se muestran contactantes a cuatro días de la elongación externa sin mediastinitis ni evidencia de fuga aérea; $\mathbf{B}$ : transoperatorio que corrobora el cabo inferior $(\mathrm{Cl})$ notablemente elongado y contactante con el cabo superior (CS); C: cabos esofágicos referidos, vascularizados y con adecuado calibre luminal posterior a la ferulación con una sonda orogástrica; D: anastomosis terminoterminal sin evidencia de tensión.

\section{CASOS CLÍNICOS}

Caso 1. Femenino, recién nacido pretérmino de 35 SDG con trisomía 21 y peso al nacer de $2,500 \mathrm{~g}$, con ausencia de permeabilidad esofágica y sialorrea. La radiografía simple de abdomen mostró ausencia de aire intestinal compatible con atresia esofágica tipo I, sin neumonitis y con persistencia de conducto arterioso sin repercusión hemodinámica (figura 1). Recibió antibióticos para tratamiento de sepsis neonatal y aspiración continua con sonda de doble lumen radiopaca marcada a nivel de cuarta vértebra torácica $\left(T_{4}\right)$, restricción hídrica y farmacología anticongestiva. Bajo mejoría de condiciones clínicas se le realizó gastrostomía tipo Stamm a los 10 días de vida y medición de cabos atrésicos con distancia radiológica de $4.5 \mathrm{~cm}$ entre la sonda de doble lumen superior y el cabo distal de $2 \mathrm{~cm}$ de longitud medido por gastrografía. A los 28 días de vida se decidió realizar por toracotomía derecha, movilización y elongación esofágica externa de ambos cabos atrésicos, encontrando un cabo esofágico inferior de 1.5 $\mathrm{cm}$, se ejecuta el procedimiento de Foker modificado (figuras 1 y 2). Al tercer día posquirúrgico se iniciaron las tracciones externas para conseguir después de cuatro días, cabos atrésicos radiopacos contactantes. A los 33 días de vida se realizó la segunda toracotomía para esofagoplastía con anastomosis sin tensión (figura 3). A las seis horas del posquirúrgico, la paciente evolucionó con hipotensión refractaria a volumen y manejo aminérgico secundario a choque cardiógeno por probable reapertura de conducto arterioso persistente. No se documentó hemotórax ni neumotórax clínico ni gastos anormales por el drenaje pleural.

Caso 2. Femenino pretérmino de 36 SDG con síndrome de Down y antecedente prenatal de polihidramnios con imagen de doble burbuja intraabdominal y probable atresia de esófago. Al nacer pesó $1,885 \mathrm{~g}$ sin permeabilidad esofágica y sialorrea, la placa simple de abdomen con ausencia de aire intestinal y sonda orogástrica de doble lumen marcada a la altura de la tercera vértebra torácica $\left(\mathrm{T}_{3}\right)$. La valoración ecocardiográfica documentó conducto arterioso permeable con boca pulmonar de $3.6 \mathrm{~mm}$, boca aórtica de $5.1 \mathrm{~mm}$ y longitud de $5.8 \mathrm{~mm}$ con presión sistólica de la arteria pulmonar de $64 \mathrm{mmHg}$ y arco aórtico a la izquierda. A los dos días de vida se le realizó laparotomía con duodenoplastía tipo Kimura 

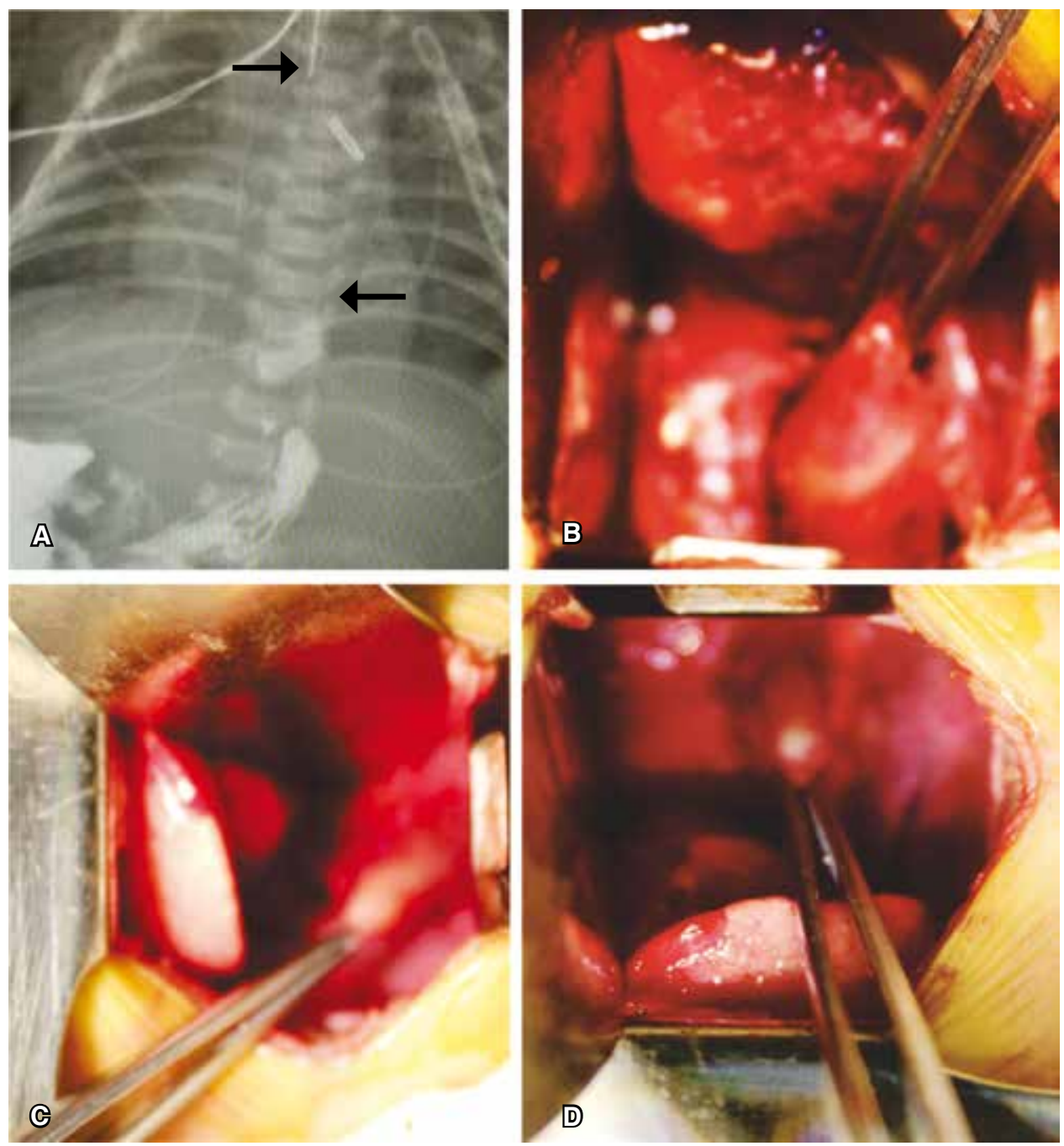

Figura 4.

A: Radiografía con sonda radiopaca de doble lumen en el cabo esofágico superior a nivel de $\mathrm{T}_{3}$ (flecha superior), el cabo inferior breve y ciego se muestra radiopaco posterior a una gastrografía (flecha inferior), compatible con atresia tipo I con una brecha amplia de 6 cuerpos vertebrales; B: disección y movilización del cabo superior; C: disección del cabo inferior de $1.3 \mathrm{~cm}$ de longitud y $0.7 \mathrm{~cm}$ de diámetro, sin comunicación aérea; y D: movilización hiatal del cabo inferior por abordaje mediastinal. por atresia duodenal con páncreas anular y gastrostomía tipo Stamm. A los 18 días de vida se efectuó cierre quirúrgico de PCA por mini toracotomía izquierda con aplicación de un hemoclip. Por gastrografía se llevó a cabo la medición de los cabos esofágicos observándose atresia esofágica tipo I con brecha amplia de seis cuerpos vertebrales entre ambos cabos, el cabo distal fue de aproximadamente $1.3 \mathrm{~cm}$ de longitud, se planeó esofagoplastía por etapas (figura 4A). El drenaje pleural se retiró a las 48 horas del cierre quirúrgico de PCA, se observó adecuada tolerancia enteral.

A los 25 días de vida con un peso de $1,870 \mathrm{~g}$, cardiopatía corregida y sin neumonitis aparente (riesgo de Spitz I), se realizó por mini toracotomía derecha procedimiento de Foker modificado encontrando: atresia de esófago tipo I con brecha amplia de $5.5 \mathrm{~cm}$ de longitud entre ambos cabos atrésicos, sin fístula a vía aérea, se practicó movilización y elongación de cabos esofágicos advirtiendo imposibilidad de anastomosis primaria con distancia de $1.5 \mathrm{~cm}$ a la máxima tracción. Se decidió referir ambos cabos para elongación extratorácica con los detalles quirúrgicos ya comentados (figuras 4 y 5 ). El proceso de elongación dio inicio a los 28 días con sedación y analgesia sin complicaciones y bajo control radiológico de aproximadamente $3 \mathrm{~mm} /$ día, hasta concebir al séptimo día del proceso cabos radiopacos contactantes. Así, se decidió realizar a los 37 días de vida una segunda toracotomía derecha con liberación de cabos atrésicos adheridos a mediastino y parénquima pulmonar y posterior esofagoplastía primaria sin tensión, se avanzó sonda orogástrica transanastomótica y se dejó drenaje pleural conectado a sello de agua y succión negativa (figura 6). Se mantuvo una semana con sedación y analgesia estrictas con apoyo ventilatorio mecánico. Un esofagograma corroboró una fuga anastomótica de la pared posterior controlada por el drenaje sin datos de mediastinitis y con adecuada expansión pulmonar de ambos hemitórax. Se decidió un manejo conservador 

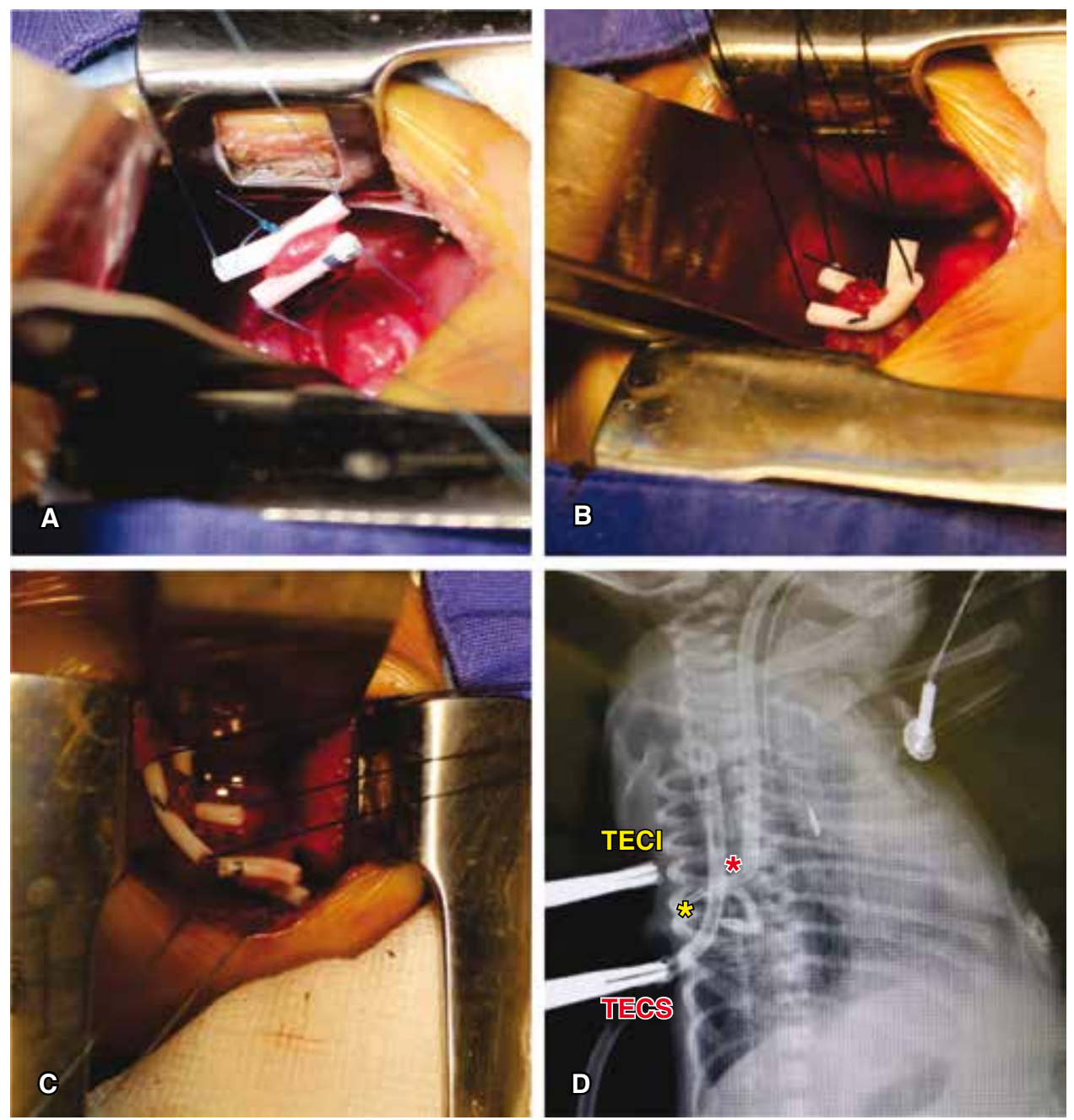

Figura 5.

A: Movilización y elongación esofágica externa aplicada al cabo inferior; B: técnica de elongación de Foker aplicado al cabo superior; $\mathbf{C}$ : ambos cabos esofágicos con una distancia de $1.5 \mathrm{~cm}$ posterior a la máxima tracción que imposibilita la anastomosis primaria; D: radiografía posoperatoria que muestra los cabos esofágicos radiopacos con una distancia de dos cuerpos vertebrales posterior a la tracción externa de los mismos, notable avance del cabo superior a juzgar por la punta de la sonda orogástrica a nivel de $T_{9}$, disminuyendo el riesgo de aspiración. hasta lograr la integridad anastomótica radiológica a los 47 días de vida (figura 7). La paciente cursó con estenosis esofágica del $30 \%$, y conscientes de los amplios factores de riesgo para aspiración por enfermedad por reflujo gastroesofágico, nuestra paciente fue sometida a cirugía antirreflujo y alimentación temporal por sonda de gastrostomía por alteración en la mecánica de deglución con evolución satisfactoria a 12 meses de evolución.

\section{DISCUSIÓN}

El interés creciente por estas técnicas de elongación esofágica ha originado extraordinarias revisiones europeas como la de Sroka et al., en donde se clasifican las estrategias quirúrgicas para el análisis de resultados y pronósticos en tres grupos, concluyendo que:

a) El grupo con la elongación esofágica de ambos cabos atrésicos de Foker provee un alto índice de anasto- mosis primarias en un promedio de tres semanas y el índice más bajo de complicaciones.

b) El grupo que combina el avance de Kimura de la esofagostomía con la técnica de Foker del cabo atrésico distal, conlleva adecuada longitud en todos los casos; pero el índice de complicaciones con infección, fugas, estenosis y anastomosis tardías es mayor.

c) El grupo con cierre de la esofagostomía previa y subsecuente técnica de Foker de ambos cabos, experimentan las complicaciones más serias: mediastinitis y perforación esofágica, infección y consecuente fallo de la anastomosis. ${ }^{6}$

Estos resultados apoyan la elongación esofágica temprana de Foker como una opción para concebir una anastomosis terminoterminal exitosa por abordajes abiertos o toracoscópicos combinados. ${ }^{2,11-14}$ Las técnicas mixtas de Kimura y Foker, aunque conllevan anas- 

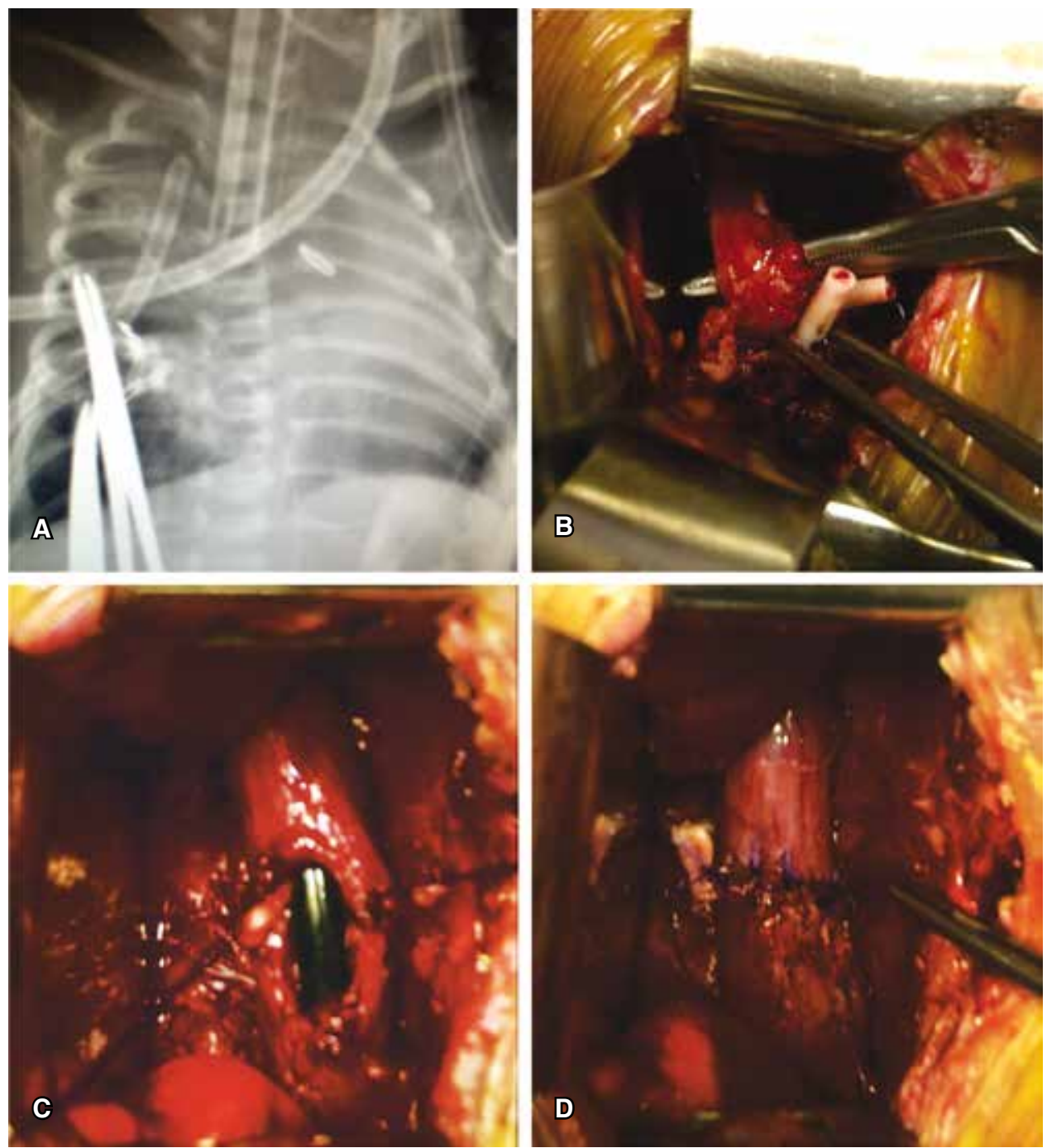

Figura 6.

A: Cabos atrésicos radiopacos que se muestran contactantes a siete días de la elongación externa; $\mathbf{B}$ : ambos cabos elongados y contactantes, la pinza de ángulo sostiene el cabo inferior y la pinza de disección refiere al cabo superior, moderada fibrosis; C: anastomosis de la pared posterior y avance de la sonda transanastomótica a través de ambos lúmenes; D: esofagoplastía temprana completada con anastomosis sin tensión. tomosis sin tensión, asocian mayores complicaciones e índice de reoperaciones. ${ }^{6,15}$ Varias técnicas dignas de mención como la elongación electromagnética de los cabos atrésicos con dilatadores metálicos por 30 a 58 días reportan anastomosis exitosas tardías con riesgos de aspiración y absceso pulmonar, perforación del cabo esofágico distal y sepsis. ${ }^{16,17}$ La elongación esofágica extratorácica descrita por Kimura e incluso combinada con la miotomía esofágica en espiral, proporciona ganancias de $3 \mathrm{~cm}$ posterior a la movilización bimensual, consiguiendo después de 1 a 5 intervenciones una anastomosis exitosa de los 5 a 24 meses de edad.5,18 Otros reportes mencionan crecimiento espontáneo de los cabos atrésicos con la aspiración continua del cabo esofágico superior durante 5 a 18 semanas para conseguir la anastomosis. ${ }^{19}$ Como acertadamente señala la revisión europea, es necesario un mayor número de registros para poder dilucidar la eficacia y seguridad de estas técnicas de elongación del esófago nativo; además, ningún paciente fue sometido a elongaciones durante la etapa neonatal (rangos de 2 a 26 meses) y las esofagoplastías exitosas se lograron en etapas tardías. Es decir, estas recomendaciones pueden ser extrapoladas, pero no aplicadas concretamente a nuestra población neonatal de estudio. Surge entonces la necesidad de elaborar una revisión de la literatura de los escasos reportes donde la elongación esofágica, en sus múltiples modalidades de inicio en el período neonatal, haya permitido una anastomosis exitosa del esófago nativo. En la tabla 1 se presentan los resultados de esta búsqueda.

El crecimiento de los segmentos esofágicos puede ocurrir durante los tres primeros meses de vida, este crecimiento inducido por tracción ha mostrado utilidad hasta los dos años, desafortunadamente estas observaciones no tienen un soporte científico o experimental 


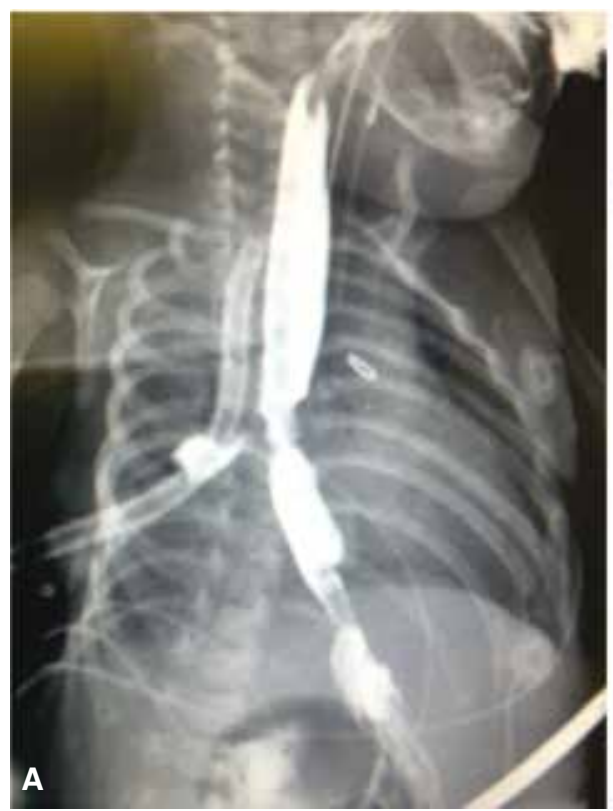

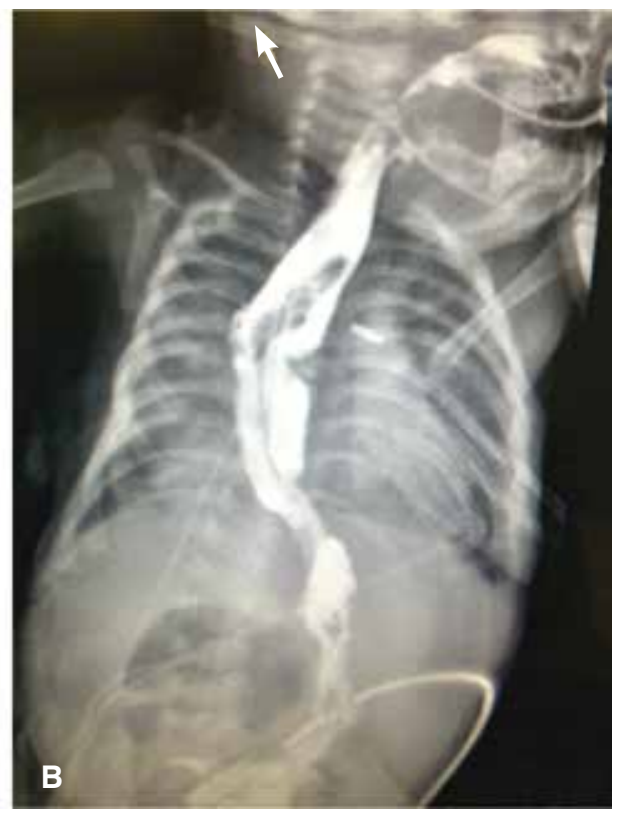

Figura 7.

A: Esofagograma que muestra a los cinco días del posoperatorio una fuga anastomótica posterior sin mediastinitis y controlada por el drenaje pleural; B: anastomosis íntegra con estenosis residual del $30 \%$ posterior a 10 días de manejo conservador. aún. ${ }^{6,10}$ Con tracciones diarias de $2 \mathrm{~mm}$ de los cabos esofágicos se han alcanzado longitudes suficientes en 6 a 18 días. $^{7}$ En nuestro caso, se logró la anastomosis a los 4 y 7 días de iniciado el proceso de elongación. Recomendamos iniciar la tracción dinámica a las 72 horas posteriores al procedimiento de Foker para permitir la granulación en el punto de sutura y disminuir el riesgo de laceración esofágica por la tracción temprana.

La técnica de elongación esofágica por tracción externa ha sido descrita por Foker desde 1997 y se ha implementado una modificación, como en el presente caso, para disminuir el riesgo de laceración y perforación esofágica del método original: sustituyendo los puntos de tracción de sutura por un solo punto transflictivo, protegiendo las suturas con tubos de silicona o polivinilo de catéteres radiopacos para su identificación y posterior evaluación. Aunque se ha descrito la disección total de los cabos esofágicos, preferimos la movilización gentil del esófago inferior a través de la disección hiatal vía mediastinal o abdominal evitando lesionar la pared y la circulación esofágica, proponiendo una gastrostomía inicial con apertura hiatal del diafragma para conseguir la medición y movilización del cabo esofágico inferior; y si las condiciones clínicas lo permiten, concebir en el mismo tiempo quirúrgico por una toracotomía mínima, el cierre de la fístula traqueoesofágica, así como la elongación y tracción esofágica externa de los cabos en aquellas atresias con imposibilidad de ejecutar la anastomosis esofágica primaria. Además, esta aproximación quirúrgica inicial con gastrostomía, cierre de fístula traqueoesofágica y procedimiento de Foker modificado, contribuyen a disminuir los factores de riesgo descritos por Spitz de elevada mortalidad para esofagoplastía primaria (neumonía por aspiración, peso $\leq 2,500 \mathrm{~g}$ y cardiopatía compleja). Los pacientes podrían requerir de una fístula sistémica pulmonar de Blalock Taussig (FBT) o el cierre quirúrgico de una persistencia de conducto arterioso si la repercusión hemodinámica de su cardiopatía congénita no permite una anastomosis definitiva exitosa. El procedimiento de Foker modificado puede representar una opción para esta conducta de esofagoplastía por etapas en los recién nacidos de alto riego, mientras se mejoran las condiciones clínicas generales para la corrección quirúrgica definitiva. Existen esofagoplastías exitosas posteriores a la técnica de Foker, incluso en aquéllas donde la dilatación con bujías o dilatadores metálicos ha fracasado.

Una propuesta flexible para todas las atresias de esófago con brecha amplia describe que si existe una tensión considerable para conseguir la anastomosis de los cabos atrésicos, se proceda a tracción interna colocando las suturas de ambos cabos elongados a la fascia prevertebral, y en 3 o 4 días se proceda a la esofagoplastía definitiva. Para las atresias con ultra long gap con imposibilidad de anastomosis, aun con la tracción intraoperatoria y donde un considerable crecimiento por elongación de los cabos es necesario, se procede a la tracción externa de Foker modificada progresando la tensión diariamente bajo control radiológico hasta considerar la anastomosis exitosa. Finalmente, una aproximación mixta se describe posterior al cierre de una fístula traqueoesofágica proximal del cabo superior; y para disminuir el riesgo de dehiscencia del cierre 
Tabla 1. Revisión de la literatura de procedimientos de elongación esofágica en atresia de esófago con brecha amplia en recién nacidos y lactantes menores de tres meses: análisis de los resultados.

\begin{tabular}{|c|c|c|c|c|c|c|}
\hline $\begin{array}{l}\text { Autor/número de } \\
\text { pacientes/edad }\end{array}$ & $\begin{array}{l}\text { Tipo de atresia/ } \\
\text { longitud del } \\
\text { long gap }(\mathrm{cm}) \text { o } \\
\text { vértebras }\end{array}$ & $\begin{array}{l}\text { Edad a la } \\
\text { primera } \\
\text { elongación }\end{array}$ & Técnica & $\begin{array}{l}\text { Número de } \\
\text { toracotomías }\end{array}$ & $\begin{array}{c}\text { Tiempo entre } \\
\text { elongación } \\
\text { y la } \\
\text { anastomosis }\end{array}$ & Complicación \\
\hline $\begin{array}{l}\text { Foker/4 } \\
\text { pacientes/no } \\
\text { se describe }\end{array}$ & $\begin{array}{l}\text { I (1), II (2), III } \\
(1) / 5.5-6.8 \mathrm{~cm}\end{array}$ & $\begin{array}{l}\text { No se } \\
\text { describe }\end{array}$ & Foker & 2 & 6 a 10 días & Estenosis esofágica \\
\hline $\begin{array}{l}\text { Al-Qahtani/3 } \\
\text { pacientes/31, } \\
34 \text { y } 37 \mathrm{SDG}\end{array}$ & $\begin{array}{l}\text { II (1), III (2)/3-5 cm } \\
\text { promedio }\end{array}$ & $\begin{array}{l}\text { Día } 2 \text { en } \\
\text { promedio }\end{array}$ & $\begin{array}{l}\text { Foker } \\
\text { modificado }\end{array}$ & 2 & $\begin{array}{l}8,12 \text { y } 15 \\
\text { días }\end{array}$ & $\begin{array}{l}\text { Quilotórax } \\
\text { Neumotórax } \\
\text { Estenosis esofágica }\end{array}$ \\
\hline $\begin{array}{l}\text { Mochizuki/2 } \\
\text { pacientes/37 } \\
\text { y } 35 \mathrm{SDG}\end{array}$ & $\begin{array}{l}\text { I (1), III (1)/> 3-4 } \\
\text { vértebras }\end{array}$ & Día 1 & $\begin{array}{l}\text { Foker } \\
\text { modificado }\end{array}$ & 2 & 5 y 14 días & Estenosis esofágica \\
\hline $\begin{array}{l}\text { Hadidi/3 } \\
\text { pacientes/32, } \\
35 \text { y } 38 \text { SDG }\end{array}$ & $\begin{array}{l}\text { I (2), III (1)/> 4-5 } \\
\text { vértebras }\end{array}$ & $\begin{array}{l}\text { No se } \\
\text { describe }\end{array}$ & $\begin{array}{l}\text { Foker } \\
\text { modificado }\end{array}$ & 2 & $\begin{array}{l}8,10 \text { y } 14 \\
\text { días }\end{array}$ & Estenosis esofágica \\
\hline $\begin{array}{l}\text { Paya/1 } \\
\text { paciente/33 } \\
\pm 4 \text { SDG }\end{array}$ & II (de Vogt)/6 cm & $\begin{array}{l}\text { No se } \\
\text { describe }\end{array}$ & Foker & 2 & 21 días & $\begin{array}{l}\text { Estenosis esofági- } \\
\text { ca resecada a } 21 / 2 \\
\text { meses }\end{array}$ \\
\hline $\begin{array}{l}\text { Van der Zee/1 } \\
\text { paciente/31 SDG }\end{array}$ & II (1)/6 vértebras & $\begin{array}{l}<2 \text { meses } \\
(2.8 \mathrm{~kg})\end{array}$ & $\begin{array}{l}\text { Foker } \\
\text { toracoscópico }\end{array}$ & $\begin{array}{l}3 \\
\text { Reoperación } \\
\text { para colo- } \\
\text { cación de } \\
\text { suturas de } \\
\text { tracción }\end{array}$ & 9 días & $\begin{array}{l}\text { Laceración esofági- } \\
\text { ca del cabo distal y } \\
\text { estenosis esofágica }\end{array}$ \\
\hline $\begin{array}{l}\text { Sroka/4 } \\
\text { pacientes/no } \\
\text { se describe }\end{array}$ & $\begin{array}{l}\text { II (2), III (2)/6 cm } \\
\text { promedio }\end{array}$ & $\leq 2$ meses & Foker & $\begin{array}{l}2 \text { en } \\
\text { promedio }\end{array}$ & $\begin{array}{l}7 \text { a } 14 \text { días; } \\
\text { uno requirió } \\
6 \text { semanas }\end{array}$ & $\begin{array}{l}\text { Estenosis y perfora- } \\
\text { ción esofágica }\end{array}$ \\
\hline $\begin{array}{l}\text { Foker } / 38 \\
\text { pacientes/2-50 } \\
\text { días }\end{array}$ & $\begin{array}{l}\text { No se describen } \\
\text { tipos/3.7 a } 12.5 \mathrm{~cm} \\
\text { promedio }\end{array}$ & $\begin{array}{l}<2 \text { meses } \\
\text { ( } 2-50 \text { días } \\
\text { promedio) }\end{array}$ & $\begin{array}{l}\text { Foker } \\
\text { a) tracción interna } \\
\text { en } 5 \\
\text { b) tracción externa } \\
\text { en } 23 \\
\text { c) tracción mixta } \\
\text { en } 10\end{array}$ & $\begin{array}{l}2 \text { en } \\
\text { promedio }\end{array}$ & $\begin{array}{l}\text { 3-4 días en } \\
\text { tracciones } \\
\text { internas } \\
\text { 8-18 días en } \\
\text { tracciones } \\
\text { externas }\end{array}$ & $\begin{array}{l}\text { Laceración de cabos } \\
\text { esofágicos } \\
\text { Adherencias y falta } \\
\text { de progreso en la } \\
\text { elongación } \\
\text { Fuga de anastomosis }\end{array}$ \\
\hline
\end{tabular}

quirúrgico de la fístula, se procede a una sola tracción interna a la fascia prevertebral y tracción externa de Foker modificado para el cabo atrésico distal. ${ }^{8}$ En nuestros reportes, el objetivo fue lograr la aproximación de los cabos atrésicos mediante la elongación esofágica para conciliar la esofagoplastía en un segundo tiempo quirúrgico y para disminuir el alto riesgo de dehiscencia por una anastomosis tensa, respectivamente. En ambos casos se logró la integridad esofágica. No obstante, la cromosomopatía y la cardiopatía de base determinaron la mortalidad en el primer caso; y en nuestro segundo reporte, fue pertinente la alimentación temporal por gastrostomía con cirugía antirreflujo para tratar la complicación de la mecánica deglutoria alterada, la estenosis y la enfermedad por reflujo gastroesofágicas asociadas. Aun si esta técnica no resulta útil para conciliar la anastomosis por etapas, la realización de una esofagostomía permitiría la ejecución del avance de Kimura para una esofagoplastía tardía, o bien algún método de sustitución esofágica.

Dentro de las complicaciones reportadas directamente al procedimiento de Foker se encuentran el quilotórax, neumotórax y laceración esofágica, las cuales son tratadas de manera conservadora junto con el drenaje pleural..$^{2,12}$ Las adherencias y la consiguiente falta de progreso en las elongaciones podrían requerir una toracotomía con adherenciolisis y recolocación de las suturas de tracción. Se ha sugerido el uso de membra- 


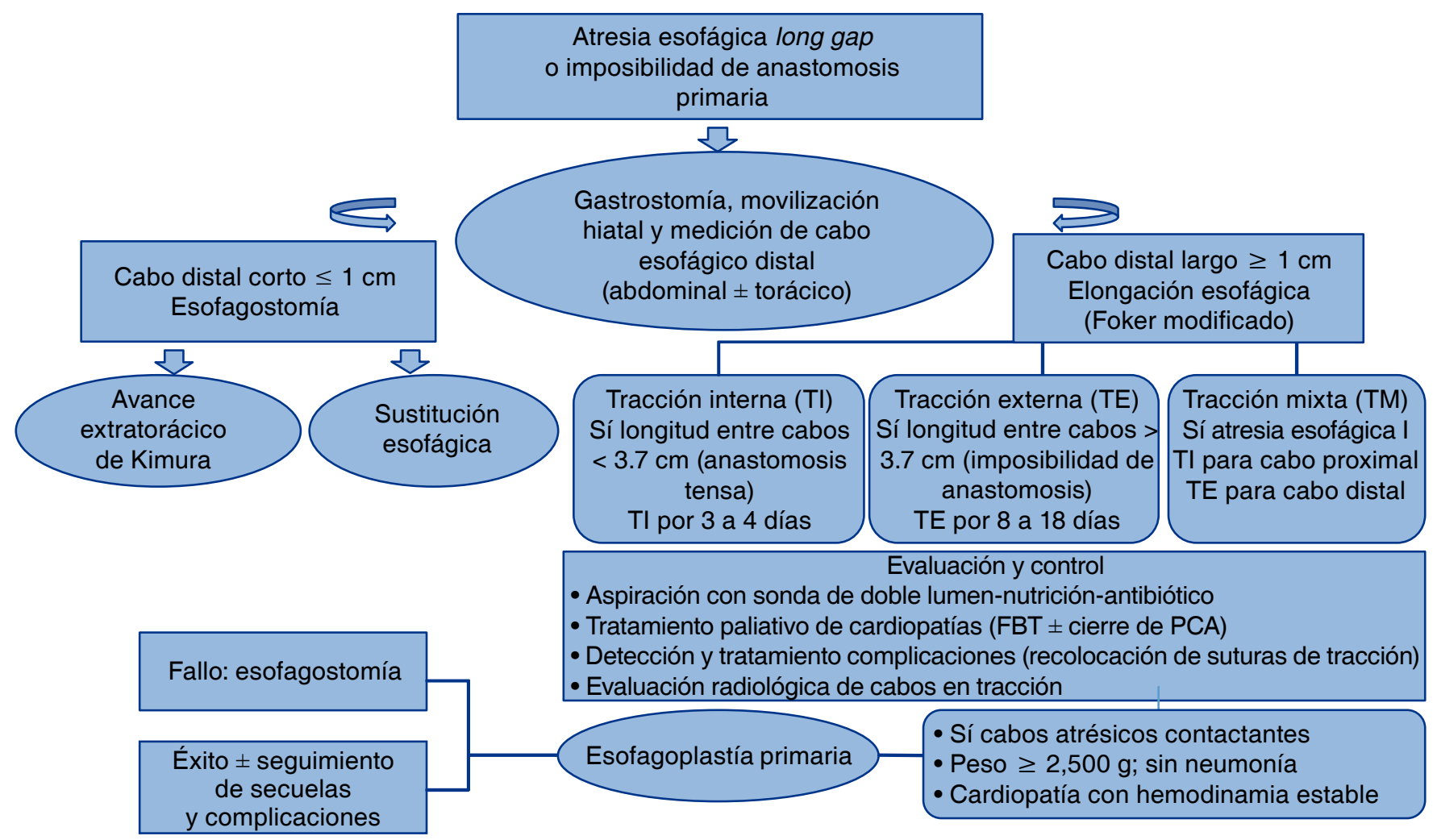

Figura 8. Propuesta de abordaje y tratamiento de la atresia de esófago con brecha amplia en el periodo neonatal.

nas quirúrgicas de Gore-Tex en los cabos sometidos a tracción para disminuir las adherencias quirúrgicas..$^{14,20}$ La estenosis esofágica y el reflujo gastroesofágico secundario, son complicaciones adyacentes a todos los procedimientos de esofagoplastía susceptibles de tratamiento conservador con dilataciones y procedimiento antirreflujo inicialmente, tal como ocurrió en nuestro segundo caso.,12,21 Se ha reportado en los pacientes sometidos a procedimiento de Foker un buen pronóstico funcional del esófago elongado en ausencia de defectos estructurales sistémicos mayores o cromosomopatías, que como en el primer caso determinan la morbimortalidad asociada. ${ }^{10}$

Una aproximación racional de la conducta quirúrgica de la atresia esofágica con brecha amplia, que considere el inicio del manejo temprano en el recién nacido y la sustitución esofágica como último recurso por su gran morbilidad asociada, propone con base en la característica de los cabos esofágicos (corto o largo) un algoritmo de actuación que se describe en la figura $8 ., 8$

En proyectos a futuro se propone la elongación esofágica de Foker mediante la vía transendoscópica de los cabos superior e inferior a través de la boca y gastrostomía, respectivamente. De esta manera po- drán ser referidos y elongados bajo la vigilancia de la toracoscopia videoasistida. Consideramos que esta propuesta de mínima invasión podría disminuir, junto con la aplicación de membranas de Gore-Tex de los cabos elongados, las adherencias y fibrosis posquirúrgicas asociadas al procedimiento original, impactando hipotéticamente en una morbilidad y tiempos quirúrgicos menores en nuestros recién nacidos de alto riesgo.

\section{REFERENCIAS}

1. Martínez-Ferro M. Atresia de esófago "long gap": definición y conducta quirúrgica moderna. Rev Med Cli Condes 2009;20:797-803.

2. Al-Qahtani AR, Yazbeck S, Rosen NG, Youssef S, Mayer SK. Lengthening technique for long gap esophageal atresia and early anastomosis. J Pediatr Surg 2003;38(5):737-739.

3. Ein SH, Shandling B, Stephens CA. Twenty one year experience with the pediatric gastric tube. J Pediatr Surg 1987;22(1):77-81.

4. Hendren WH, Hendren WG. Colon interposition for esophagus in children. J Pediatr Surg 1985;20(6):829-839.

5. Kimura K, Nishijima E, Tsugawa C, et al. Multistaged extrathoracic esophageal elongation procedure for long gap esophageal atresia: experience with 12 patients. J Pediatr Surg 2001;36(11):1725-1727. 
6. Sroka M, Wachowiak R, Losin M, et al. The Foker technique (FT) and Kimura advancement (KA) for the treatment of children with long-gap esophageal atresia (LGES): lessons learned at two European centers. Eur J Pediatr Surg 2013;23(1):3-7. doi: 10.1055/s-0033-1333891.

7. Foker JE, Linden BC, Boyle EM Jr, Marquardt C. Development of a true primary repair for the full spectrum of esophageal atresia. Ann Surg 1997;226(4):533-541.

8. Foker JE, Kendall TC, Catton K, Khan KM. A flexible approach to archieve a true primary repair for all infants with esophageal atresia. Semin Pediatr Surg 2005;14(1):8-15.

9. Paya K, Schlaff N, Pollak A. Isolated ultra-long gap esophageal atresia- successful use of the Foker technique. Eur J Pediatr Surg 2007;17(4):278-281.

10. Foker JE, Kendall Krosch TC, Catton K, Munro F, Khan KM. Long-gap esophageal atresia treated by growth induction: the biological potential and early followup results. Semin Pediatr Surg 2009;18(1):23-29. doi: 10.1053/j.sempedsurg.2008.10.005.

11. Mochizuki K, Obatake M, Taura $\mathrm{Y}$, et al. A modified Foker's technique for long gap esophageal atresia. Pediatr Surg Int 2012;28(8):851-854. doi: 10.1007/s00383012-3151-1.

12. Hadidi AT, Hosie S, Waag KL. Long gap esophageal atresia: lengthening technique and primary anastomosis. J Pediatr Surg 2007;42(10):1659-1662.

13. van der Zee DC. Thoracoscopic elongation of the esophagus in long gap esophageal atresia. J Pediatr Gastroenterol Nutr 2011;52 Suppl 1:S13-S15. doi: 10.1097/ MPG.0b013e3182125d75.

14. van der Zee DC, Vieirra-Travassos D, Kramer WL, Tytgat SH. Thoracoscopic elongation of the esophagus in long gap esophageal atresia. J Pediatr Surg 2007;42(10):1785-1788.
15. Till H, Rolle U, Siekmeyer W, Hirsch W, Foker J. Combination of spit fistula advancement and external traction for primary repair of long-gap esophageal atresia. Ann Thorac Surg 2008;86(6):1969-1971. doi: 10.1016/j.athoracsur.2008.05.056.

16. Hendren WH, Hale JR. Esophageal atresia treated by electromagnetic bougienage and subsequent repair. J Pediatr Surg 1976;11(5):713-722.

17. Hendren WH, Hale JR. Electromagnetic bougienage to lengthen esophageal segments in congenital esophageal atresia. N Engl J Med 1975;293(9):428-432.

18. Takamizawa S, Nishijima E, Tsugawa C, et al. Multistaged esophageal elongation technique for long gap esophageal atresia: experience with 7 cases at a single institution. J Pediatr Surg 2005;40(5):781-784.

19. Puri P, Khurana S. Delayed primary esophageal anastomosis for pure esophageal atresia. Semin Pediatr Surg 1998;7(2):126-129.

20. Dessanti A, Caccia G, lannuccelli M, Dettori G. Use of "Gore-Tex surgical membrane" to minimize surgical adhesions in multistaged extrathoracic esophageal elongation for esophageal atresia. J Pediatr Surg 2000;35(4):610-612.

21. Konkin DE, O'hali WA, Webber EM, Blair GK. Outcomes in esophageal atresia and tracheoesophageal fistula. J Pediatr Surg 2003;38(12):1726-1729.

\section{$\triangle$ Correspondencia:}

Dr. Rogelio Sancho-Hernández Instituto Nacional de Pediatría. Insurgentes Sur Núm. 3700-C, Colonia Insurgentes Cuicuilco. México, D.F. Correo electrónico: saherog@yahoo.com.mx

Los autores declaran no tener conflicto de intereses. 